\title{
Surface Modification of Polypropylene Membrane Using Biopolymers with Potential Applications for Metal Ion Removal
}

\author{
Omar Alberto Hernández-Aguirre, ${ }^{1}$ Alejandra Núñez-Pineda, ${ }^{2}$ \\ Melina Tapia-Tapia, ${ }^{2}$ and Rosa María Gómez Espinosa ${ }^{1}$ \\ ${ }^{1}$ Facultad de Química, UAEM and Centro Conjunto de Investigación en Química Sustentable UAEM-UNAM,
km 14.5 Carretera Toluca-Atlacomulco, San Cayetano, 50200 Toluca, MEX, Mexico
${ }^{2}$ Instituto de Química, UNAM and Centro Conjunto de Investigación en Química Sustentable UAEM-UNAM,
km 14.5 Carretera Toluca-Atlacomulco, San Cayetano, 50200 Toluca, MEX, Mexico
}

Correspondence should be addressed to Rosa María Gómez Espinosa; rosamarigo@gmail.com

Received 7 April 2016; Revised 2 June 2016; Accepted 7 June 2016

Academic Editor: João Paulo Leal

Copyright (C) 2016 Omar Alberto Hernández-Aguirre et al. This is an open access article distributed under the Creative Commons Attribution License, which permits unrestricted use, distribution, and reproduction in any medium, provided the original work is properly cited.

\begin{abstract}
This work aims to present the modification of polypropylene (PP) membranes using three different biopolymers, chitosan (CHI), potato starch (PS), and cellulose (CEL), in order to obtain three new materials. The modified membranes may be degraded easier than polypropylene ones and could be used as selective membranes for metal ions removal, among other applications. For this purpose, the UV energy induced graft copolymerization reaction among polypropylene membrane, acrylic acid, benzophenone (as photoinitiator), and the biopolymer (CHI, PS, or CEL) was conducted. The results of FT-IR-ATR, XRD, TGA, DSC, SEM, BET, and AFM analyses and mechanical properties clearly indicate the successful modification of the membrane surface. The change of surface wettability was monitored by contact angle. The grafting reaction depends on natural polymer, reaction time, and concentration. In order to prove the potential application of the modified membranes, a preliminary study of sorption of metal ion was carried out. For this purpose, the PP-CHI membrane was chosen because of the high hydrophilicity, proportionate to - $\mathrm{OH}$ and $\mathrm{NH}_{2}$; these groups could act as ligands of metal ions, provoking the interaction between PP-CHI and $\mathrm{M}+\left(\mathrm{PP}-\mathrm{CHI}-\mathrm{M}^{+}\right)$and therefore the metal ion removal from water.
\end{abstract}

\section{Introduction}

Polypropylene (PP) is widely used in a variety of industrial applications such as wastewater treatment and separation process because of its low cost, good mechanical and thermal properties, and chemical stability. In the membrane field, PP is also considered for biomedical applications, namely, hemodialysis, plasmapheresis, blood oxygenation, and leukodepletion process [1]. However, the hydrophobicity of PP material limits its applications and consequently many compounds have been used to modify the PP surface [2-4], in order to change its properties and increase their applications. The surface of PP films has been grafted with polar groups [5, 6] to modify its permeability coefficient and hydrophilicity. Grafting modification with specific functional groups is one of the approaches to synthesize adsorbent PP that can be used for metal removal from wastewater [7], where the selectivity depends on the specific interactions between the grafted monomer and the metal ions.

The surface modification of membranes is a promising approach to confer new properties to the membranes, different from those of the original material. Surface chemistry and morphology of membranes play an important role in the efficiency of the membrane modification process [8]. Generally, the objective of the modification is not only to increase the flux and/or selectivity, but also to improve the chemical resistance [9]. A monomer could be introduced within a polymeric matrix using blend or immobilization processes, such as other several ways [10]. However, the compatibility of the monomer with the polymeric matrix becomes a prime constraint to develop the required material [11]. In this context, several methods have been used to 
modify the chemistry of membranes. These include surface grafting [12], pore-filled grafting [13], and dip coating [14]. The UV-induced graft polymerization of a monomer into the polymer matrix is an adequate route to modify the surface; it changes its properties such as wettability or permeability. The attractive feature of the grafting process is that the extent of modification can be precisely controlled by the proper selection of the irradiation and reaction conditions [10]. In addition, the graft polymerization is initiating without prior modification of a surface. The procedure is relatively simple, energy-efficient, cost-effective, and well-suited for integration with other technologies [15]. The matrix can be modified with different functional groups such as carboxylic acid, amine, hydroxyl, and sulfonic acid groups. These groups attached onto the polymeric networks can be tailored for a specific application [16]. Owing to higher adsorption rate and adsorption capacity, the modified polymer can provide many advantages as new-type, fast-responsive, and highcapacity adsorbent materials for the removal of pollutants from aqueous solutions $[17,18]$.

Biopolymers are good candidates to modify the surface properties of PP, due to their properties such as water binding capacity, nontoxicity, biocompatibility, selectivity, and biodegradability. Therefore, it is reasonable to combine the advantages of UV-induced graft polymerization with the versatile characteristics of natural polymers like potato starch (PS), cellulose (CEL), and chitosan (CHI), which occur abundantly in nature [19-22]. The present work is concerned with the use of the natural polymers as grafting compounds to modify PP using UV energy source to obtain new membranes with capabilities of water affinity, metal removal, and potential biodegradability after use. It was decided to functionalize the polypropylene membrane with acrylic acid, taking advantage of the polar groups $(\mathrm{C}=\mathrm{O},-\mathrm{OH})$ to anchor the biopolymer (Figure 1).

\section{Materials and Methods}

Polypropylene microporous membrane with pore size of $0.45 \mu \mathrm{m}$, thickness of $114 \mu \mathrm{m}$, and porosity of $84.6 \%$ was supplied by $3 \mathrm{M}$ Company. Chitosan (448877-medium molecular weight, deacetylated chitin), cellulose ( $\alpha$-cellulose), phosphoric acid, acrylic acid, and benzophenone (radical photoinitiator) were from Sigma-Aldrich and were used without prior treatment. Potato starch was obtained by the method reported by BeMiller and Whistler [19].

2.1. Preparation of Biopolymers Solutions for Membrane Surface Modification. Solutions of $1 \% \mathrm{w} / \mathrm{v}$ of each biopolymer were prepared: potato starch (in water), chitosan (in $2 \%$ acetic acid solution), and cellulose (in phosphoric acid). In addition, solutions of acrylic acid (1:1 in deionized water) and benzophenone $(0.05 \mathrm{M}$ in acetone) were also prepared.

2.2. Grafting of Biopolymers into Polypropylene Surface. The polypropylene membrane was cut into square-shaped pieces $(5 \times 5 \mathrm{~cm})$ and impregnated with corresponding biopolymer solution, acrylic acid, and benzophenone. The so-prepared membranes were put in between two liners, placed in squeeze rollers, and transferred to a glass plate and another glass plate was put on top of it. Membranes were irradiated with UV radiation $(300 \mathrm{~nm})$ during $8 \mathrm{~h}$. After this time, the samples were washed in Soxhlet equipment for $24 \mathrm{~h}$ to remove nongrafted biopolymer. The grafted membranes were dried under vacuum until constant weight.

\subsection{Graft Polymerization for Kinetics Analysis}

2.3.1. Determination of Grafting Degree. The grafting degree (Gd) was calculated from the equation

$$
\operatorname{Gd}(\%)=\frac{\left(W_{1}-W_{0}\right)}{W_{0}} \times 100,
$$

where $W_{0}$ and $W_{1}$ are the mass of the PP membrane and the modified membrane, respectively.

2.3.2. Effect of the Irradiation Time. The effect of irradiation time was evaluated by the grafting degree determined at 1 to 8 hours of exposure to UV energy.

2.3.3. Effect of the Biopolymer Concentration. The effect of biopolymer concentration was evaluated at $0.25,0.5,1$, and $2 \%$ in solution.

2.4. Methods of Characterization. The modified membranes of polypropylene have been characterized using the following methods.

2.4.1. Wettability and Contact Angle Test. To determine the equilibrium water absorption, the modified membranes were subjected to swelling test. The samples were immersed in deionized water during different periods. Excess of water on the surface of the samples was removed with filter paper and the swollen samples were weighted. The swelling was determined by the following equation:

$$
\text { Swelling }(\%)=\frac{\left(W_{s}-W_{i}\right)}{W_{i}} \times 100,
$$

where $W_{s}$ and $W_{i}$ are the weights of swollen and dry membranes, respectively.

Contact angle measurements were conducted in a Krüss model G-1 apparatus.

2.4.2. Fourier Transform Infrared Spectroscopy (FT-IR). FTIR-ATR spectra were acquired by an infrared spectrometer Bruker Tensor 27; the ATR accessory contained a diamond crystal. The spectra acquisition conditions were $25^{\circ} \mathrm{C}$, 64 scans, and $500-4000 \mathrm{~cm}^{-1}$ and $2 \mathrm{~cm}^{-1}$ resolution, with respect to the appropriate background spectra.

2.4.3. Scanning Electronic Microscopy (SEM). Scanning Electronic Microscopy was performed in JEOL JSM-6510LV equipment. For this analysis the membranes were first dried until constant weight and then covered with gold to improve images quality. 

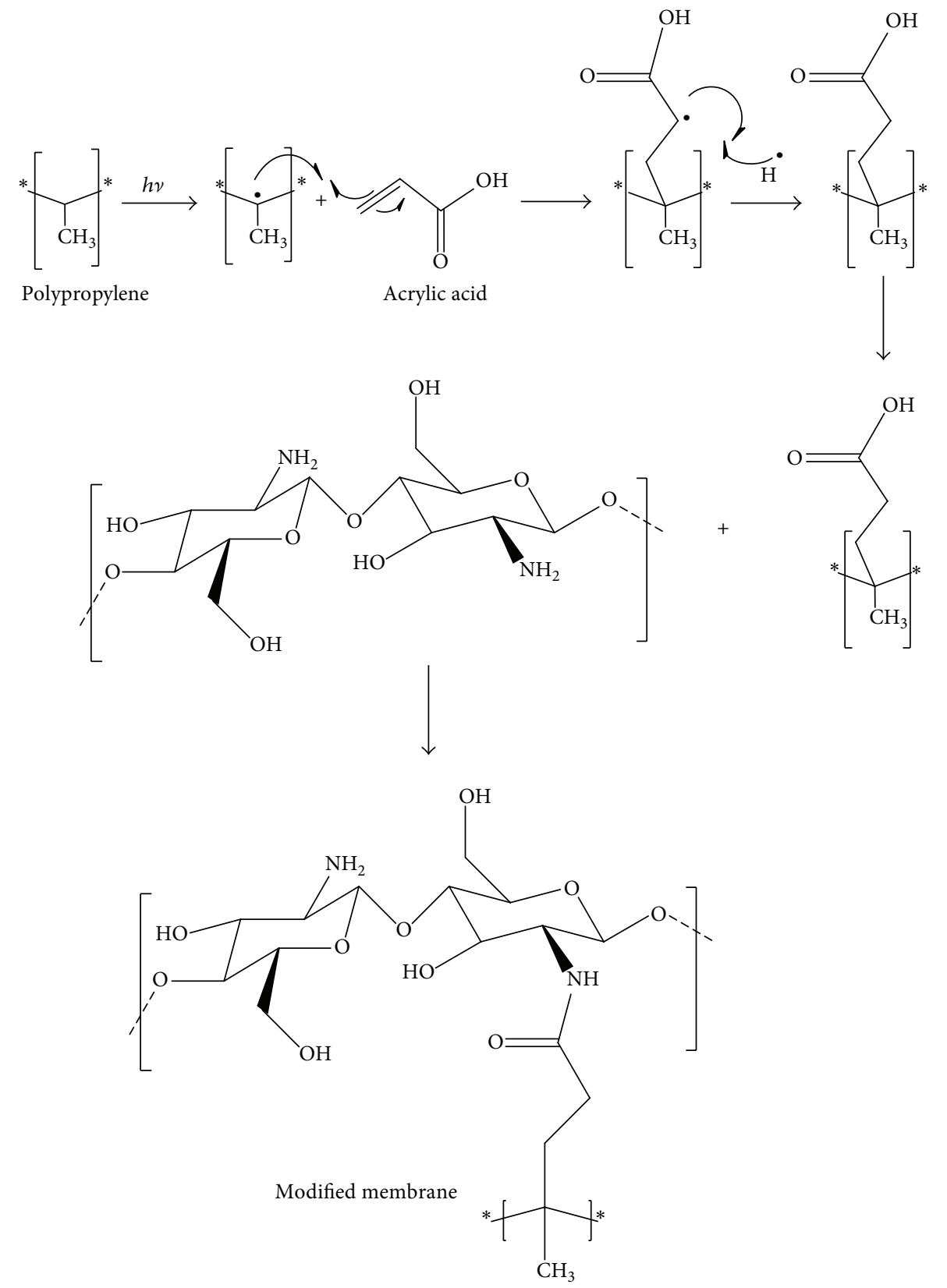

FIGURE 1: Schematic proposal of grafting of each biopolymer on the surface of PP membrane using radiation UV. (Chitosan was used for this example.)

2.4.4. Atomic Force Microscopy (AFM). AFM was performed with the noncontact mode using a MFP-3D Origin AFM apparatus equipped with Igor Pro 6.34A. The sample was attached to a glass slide using a double-sided tape, followed by scanning of the surface by a silicon nitride probe in air at ambient conditions. The scanning was performed at a speed of $0.24 \mathrm{~Hz}$ and scan sizes of $90,60,30$, and $15 \mu \mathrm{m}$. Sampling resolution of 448 points per line was selected.

2.4.5. Thermogravimetric Analysis (TGA) and Differential Scanning Calorimetry (DSC). TGA and DSC studies were carried out in a thermal analyzer Netzsch STA 449 F3 Jupiter, with a heating ramp of $10^{\circ} \mathrm{C} / \mathrm{min}$, in a nitrogen atmosphere at a flow rate of $20 \mathrm{~mL} / \mathrm{min}$. Samples were heated from room temperature to $560^{\circ} \mathrm{C}$. Aluminum crucibles of $5 \mathrm{~mm}$ diameter were used. Savitzky-Golay's smoothing algorithm was employed for TGA curves.

2.4.6. Specific Surface Area (Using BET). The specific surface area of the modified membrane was determined by $\mathrm{N}_{2}$ adsorption using the multipoint Brunauer-Emmett-Teller isotherm (BET) in Autosorb iQ equipment of Quantachrome Instruments at $77 \mathrm{~K}$. 


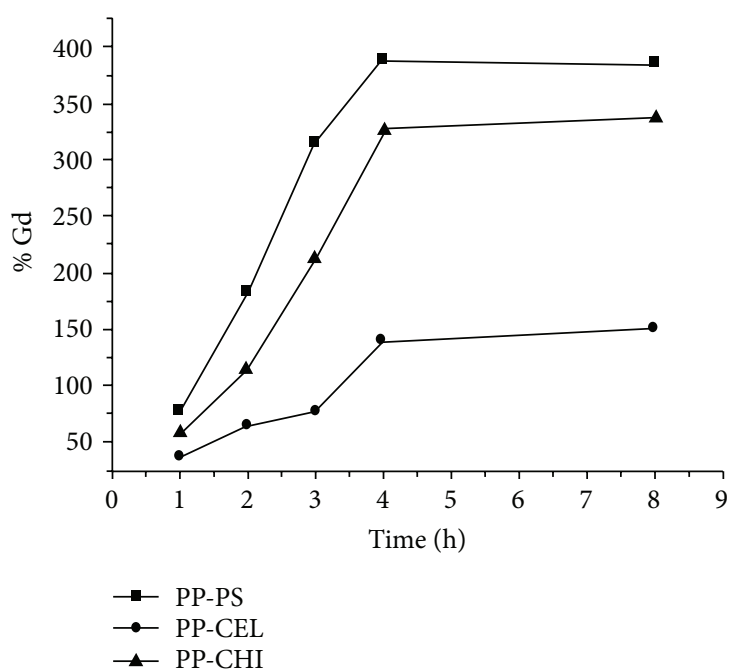

(a)

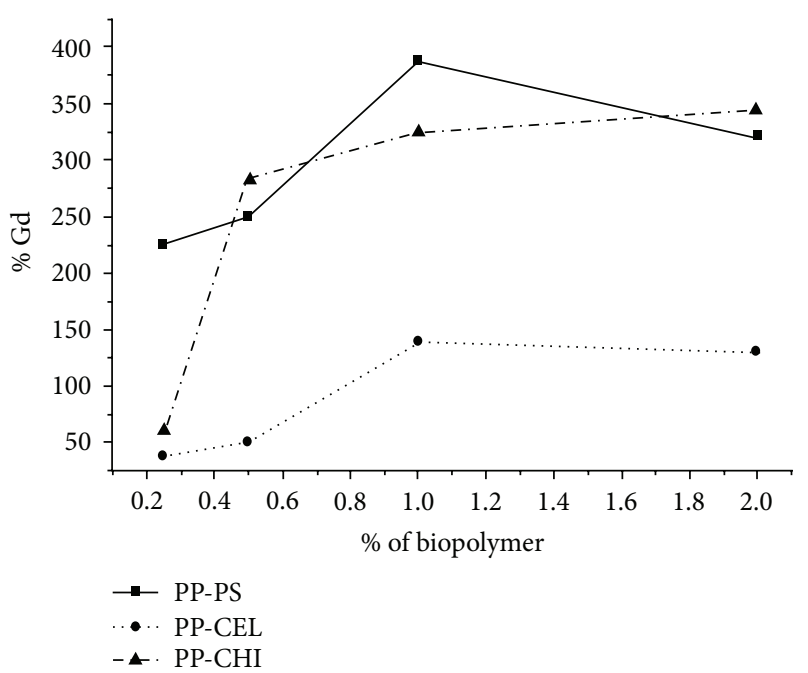

(b)

Figure 2: (a) Grafting yield of biopolymer as function of the reaction time. (b) Effect of biopolymer concentration on grafting degree.

2.4.7. X-Ray Diffraction (XRD). The diffraction patterns were obtained using Bruker D8 Advance, Cu-k $\alpha$ to $1.5404 \AA$ at $2 \theta\left(5-60^{\circ}\right)$ at $30 \mathrm{kV}$ and $20 \mathrm{~mA}$. The scanning electron microscopy SEM-EDS was performed in JEOL JSM-6510LV. Elemental analysis was performed in an Elementar Vario Micro Cube analyzer.

2.4.8. Mechanical Test. Tensile modulus and ultimate loadto-failure strength were determined on separate strips of $1 \times$ $5 \mathrm{~cm}$ by a tensile test in TA Instruments DMA-Q800.

2.5. Sorption Test. A sorption test was conducted with a variable flow peristaltic pump (Pump Low Speed Flow Control Company), latex connections, a $20 \mathrm{mg} / \mathrm{L}$ copper solution prepared from copper pentahydrate sulfate $\left(\mathrm{CuSO}_{4} * 5 \mathrm{H}_{2} \mathrm{O}\right)$, and deionized water (DI). The system was conditioned at a flow rate of $2 \mathrm{~mL} / \mathrm{min}$.

\section{Results and Discussion}

3.1. Grafting of Biopolymers onto Polypropylene Surface. The reaction was carried out by free radical polymerization of acrylic acid, benzophenone (as photoinitiator), and biopolymers (CEL, PS, and CHI, separately). In previous experiments by our group, the need of acrylic acid addition to achieve the biopolymers grafting has been established. The reaction results in the formation of the interpolymer network where biopolymer molecules are grafted into the macromolecular lattice acrylic acid-polypropylene [25, 26]. Thus, once the $\mathrm{PP}$ was functionalized, the modification using biopolymer is feasible.

3.1.1. Determination of Grafting Degree, Concentration, and Irradiation Time. The dependence of the reaction time and biopolymer concentration on the grafting degree was evidenced in Figure 2. It is worth pointing out that the acrylic acid provides the reactive sites for the grafting of the biopolymer; otherwise the reaction does not occur [27]. In Figure 2(a) the graft copolymerization is proportional to the reaction time, where the modified membranes presented the highest grafting yields at 4 hours. The observed behavior for the grafting yield of the biopolymers was as follows: PS (388\%), CHI (240\%), and CEL (140\%). Figure 2(b) shows the dependence of the grafting degree on biopolymer concentration. The grafting yield of the reaction system could change with the different reactant concentrations. It can be seen that an increase of the biopolymer concentration leads to a Gd enhancement. However, the biopolymer concentration increased to a maximum of $1 \%$ and then decreased. The initial positive slope of the plot is explained by an increase of the grafting reaction due to a greater concentration of the reactant. When the biopolymer concentration further increased, an increase in the viscosity of the grafting mixture was observed producing a difficult diffusion of the biopolymer into the PP $[25,27]$. It is often reported that the grafting efficiency increases with monomer concentration up to a certain limit and then decreases with higher concentrations [28-30]. These results indicated that the optimal conditions for this study were 4 hours of reaction time and $1 \%$ of the biopolymer concentration to modify polypropylene surface.

3.2. Characterization of Modified Membranes. To evaluate the hydrophilic character of the modified membrane, the contact angle and the kinetics of swelling were studied.

3.2.1. Wettability Test of Membrane. The kinetics of swelling of the modified membrane at room temperature in water was followed by gravimetry. Figure 3 shows the swelling process of each modified membrane. The equilibrium was observed after 20 minutes for each new membrane with a maximum absorption as follows: PP-PS (247\%), then PP-CEL (177\%), and PP-CHI (164\%). The unmodified polypropylene 


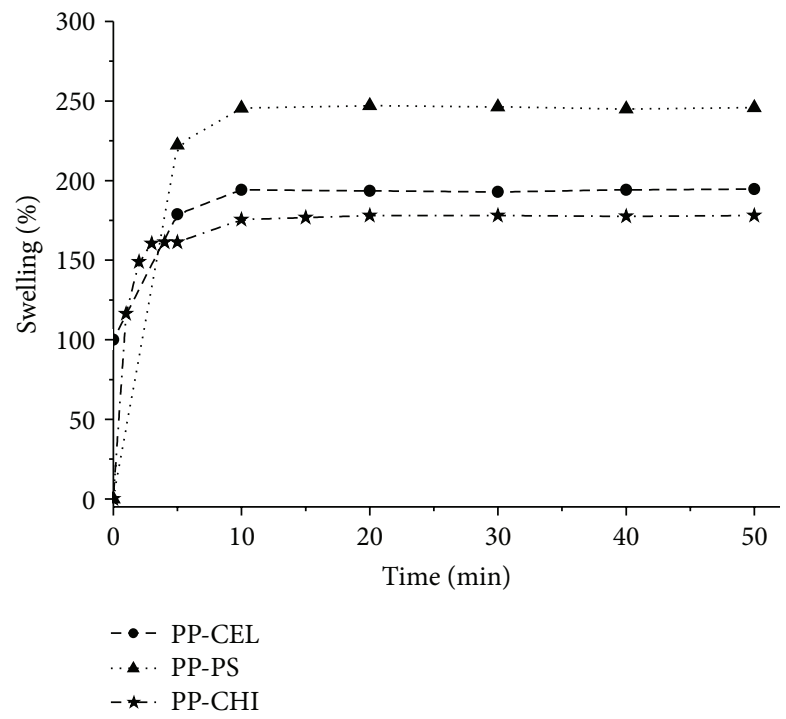

FIgURE 3: Swelling process of modified membranes.

membrane did not present swelling ability. The grafted polar groups provoked a radical change in the hydrophobic character of the membrane $[31,32]$.

3.2.2. Contact Angle. The interfacial properties between a liquid and a polymer component are characterized by the surface energies of each phase and the contact angle between them [33]. Contact angle measurements have been commonly used to characterize the hydrophilicity of membrane surfaces [15]; the unmodified membrane had a $90^{\circ}$ water contact angle, while modified membranes decreased the angle depending on the grafted biopolymer on PP surface. The PP-CEL membrane showed an angle of $25^{\circ}$; the PP-CHI membrane presented $16^{\circ}$; when it comes to the PP-PS, the contact angle was $0^{\circ}$, and this indicates a dramatic change in its hydrophobic character, having complete wetting. These results show that the modified membranes changed the hydrophobicity of the polypropylene membrane, due to the presence of polar groups from the biopolymers, which enhances the surface energy and the hydrophilicity of modified membranes [34]. The hydrophilicity will influence the permeation and surface properties of the PP membranes, thus widening their applications in biomedical and environmental fields [35].

3.2.3. FT-IR-ATR Analysis. The FT-IR spectroscopy can confirm that each biopolymer was grafted onto the PP membrane surface. Figure 4 shows the FT-IR-ATR analysis of modified membranes compared with PP, as reference. The PP spectrum shows the absorbency peaks at $2918 \mathrm{~cm}^{-1}\left(\mathrm{CH}_{2}\right.$ stretching asymmetrical vibration), $2848 \mathrm{~cm}^{-1}\left(\mathrm{CH}_{2}\right.$ stretching symmetrical vibration), and $1455 \mathrm{~cm}^{-1}$ and $1380 \mathrm{~cm}^{-1}\left(\mathrm{CH}_{2}\right.$ scissor vibration), and other low frequency -C-C- stretching vibration modes are also observed. The spectra of modified membranes PP-PS and PP-CEL showed characteristic signals at 3159 and $3367 \mathrm{~cm}^{-1}$ corresponding at stretching
TABLE 1: Elemental analysis of modified membranes.

\begin{tabular}{lccccc}
\hline Membrane & \% Gd & Carbon & Hydrogen & Oxygen & Nitrogen \\
\hline PP & - & 85.50 & 13.92 & - & - \\
PP-PS & 388 & 67.68 & 10.14 & 22.18 & - \\
PP-CEL & 140 & 68.24 & 10.68 & 21.08 & - \\
PP-CHI & 240 & 54.55 & 7.64 & 37.7 & 0.11 \\
\hline
\end{tabular}

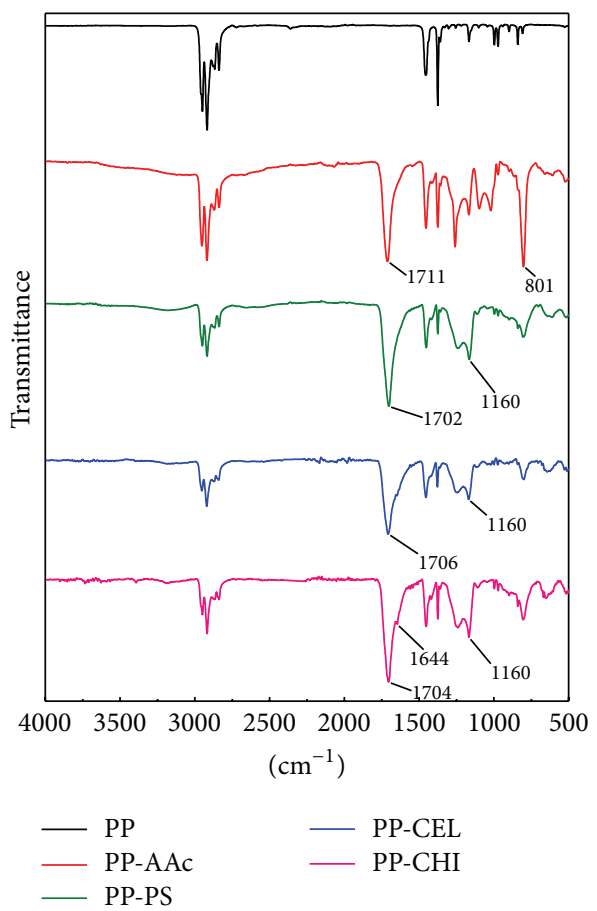

FIGURE 4: FT-IR-ATR spectra of modified membranes (PP-CHI, PP$\mathrm{CEL}$, and PP-PS) and PP.

vibration of $-\mathrm{OH}$, respectively. $\mathrm{PP}-\mathrm{CHI}$ spectrum showed a broad band at $3161 \mathrm{~cm}^{-1}$ of stretching vibration of $\mathrm{N}-\mathrm{H}$ and $\mathrm{O}-\mathrm{H}$; the signal in $1644 \mathrm{~cm}^{-1}$ corroborates the bending vibration of $\mathrm{N}-\mathrm{H}$. The $\mathrm{C}=\mathrm{O}$ vibration had a different shift depending on the biopolymer grafted; for example, the PPPS was observed at $1702 \mathrm{~cm}^{-1}$, the PP-CEL at $1706 \mathrm{~cm}^{-1}$, and the PP-CHI at $1704 \mathrm{~cm}^{-1}$. The spectra of PP-PS, PPCEL, and PP-CHI showed the same pattern around 1200$1160 \mathrm{~cm}^{-1}$ corresponding to the glycosidic bond (C-O-C stretching vibration) of the biopolymers. It is important to mention that the carbonyl group of the acrylic acid grafted to polypropylene membrane (PP-AAc) showed a different shift at $1711 \mathrm{~cm}^{-1}$.

3.2.4. Elemental Analysis of the Modified Membranes. This analysis showed that the results are consistent with the empirical equation of $\mathrm{PP}\left(\mathrm{C}_{3} \mathrm{H}_{6}\right)$ that is calculated as $85.71 \%$ of carbon and $14.29 \%$ of hydrogen. According to the elemental analysis results for PP-PS, PP-CEL, and PP-CHI (Table 1), there is a significant amount of oxygen caused by the presence of acrylic acid and biopolymer. The analyses of the composition of the modified membrane of PP-PS and 


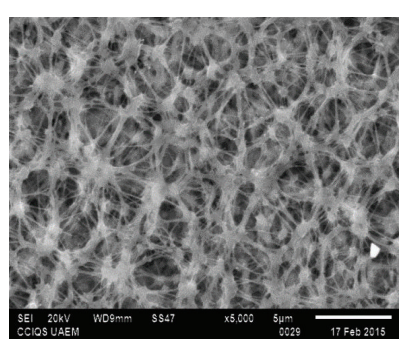

(a)

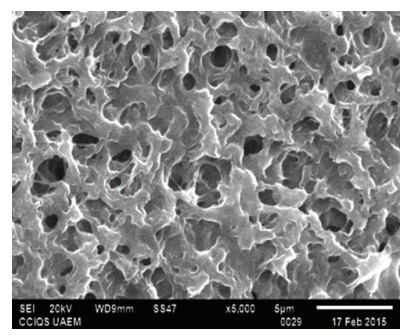

(c)

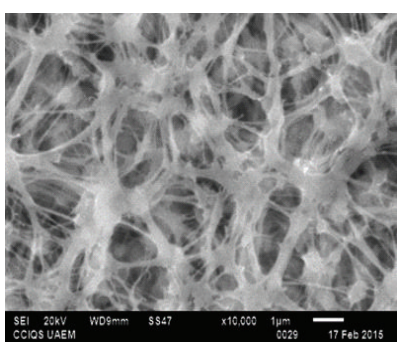

$\left(a^{\prime}\right)$

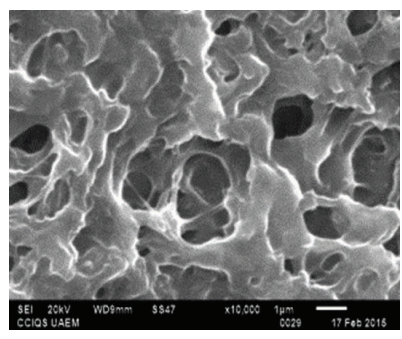

$\left(c^{\prime}\right)$

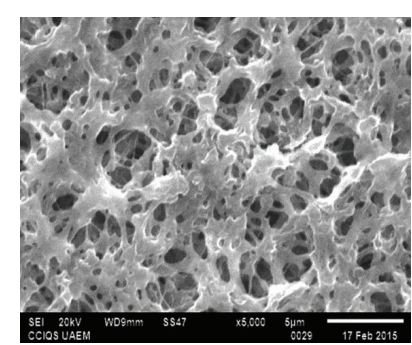

(b)

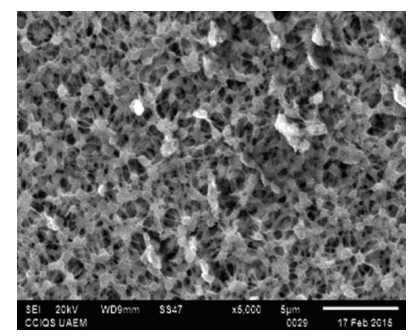

(d)

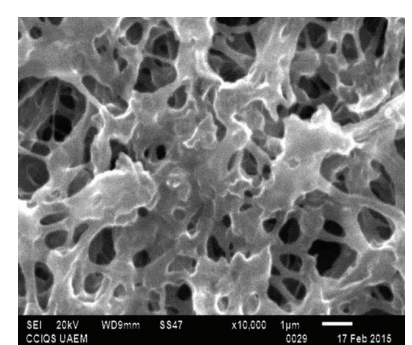

$\left(b^{\prime}\right)$

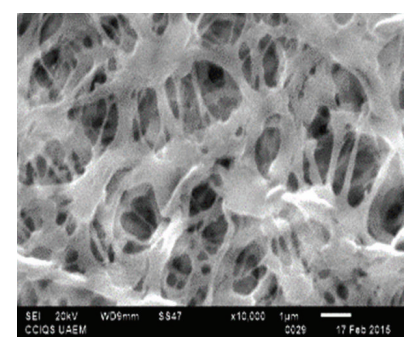

$\left(d^{\prime}\right)$

FIGURE 5: SEM images of membranes: unmodified PP, (a) $\times 5000$ and $\left(\mathrm{a}^{\prime}\right) \times 10000$; PP-PS, (b) $\times 5000$ and $\left(\mathrm{b}^{\prime}\right) \times 10000$; PP-CEL, (c) $\times 5000$ and $\left(c^{\prime}\right) \times 10000$; and PP-CHI, (d) $\times 5000$ and $\left(d^{\prime}\right) \times 10000$.

PP-CEL have the same empirical equation $\left(\mathrm{C}_{6} \mathrm{H}_{10} \mathrm{O}_{5}\right)$, unlike chitosan $\left(\mathrm{C}_{6} \mathrm{H}_{11} \mathrm{O}_{4} \mathrm{~N}\right)$ that showed the presence of nitrogen from the amine group. These results are similar to the sum of PP + AAc + biopolymer, for example. In the case of $\mathrm{PP}-\mathrm{CHI}$ the theoretical composition was $52.36 \%$ of carbon, $7.6 \%$ of hydrogen, $32.98 \%$ of oxygen, and $5.1 \%$ of nitrogen for $\mathrm{C}_{12} \mathrm{H}_{21} \mathrm{O}_{6} \mathrm{~N}$. Certainly, these results clearly indicate the presence of the biopolymer in the PP grafted membrane.

3.2.5. Scanning Electron Microscopy (SEM). Scanning electron microscopy (SEM) was used to examine the morphology of the different modified membranes. It can be observed in Figure 5 that the surface of the modified membranes is considerably different from the starting membrane. The PP unmodified membrane (Figures $5(\mathrm{a})$ and $5\left(\mathrm{a}^{\prime}\right)$ ) shows well-defined fibers forming pores of heterogeneous size. The changes in the morphology are evident depending on the grafted biopolymer, observing a greater increase in the thickness of the fibers in the modified membrane: PPPS (Figures $5(\mathrm{~b})$ and $5\left(\mathrm{~b}^{\prime}\right)$ ), followed by PP-CEL (Figures $5(\mathrm{c})$ and $5\left(\mathrm{c}^{\prime}\right)$ ), and PP-CHI (Figures $5(\mathrm{~d})$ and $5\left(\mathrm{~d}^{\prime}\right)$ ). We attributed the different thickness to the chemical structure of each biopolymer and to their different molecular weight.

3.2.6. BET Assay. The BET analysis was used as a complementary assay. The specific surface area of the modified membrane was determined by the physical adsorption of nitrogen on the surface of the membrane and by calculating the amount of adsorbate gas corresponding to a layer on the surface. The results show that the addition of the biopolymer reduces the total area and the pore diameter. This is in agreement with the SEM images. The diminished porosity of the modified membrane can be ascribed to the biopolymer grafted in the surface, causing more resistance to the lamellar separation [34]. The BET surface area of the PP membrane was $922.3 \mathrm{~m}^{2} / \mathrm{g}$ and is characteristic of a microporous material. However, the modified membranes exhibit surface areas in the following order: PP-CHI > PPCEL $>$ PP-PS $\left(48.6,46.6\right.$, and $42.6 \mathrm{~m}^{2} / \mathrm{g}$, resp.). This indicates that the biopolymer has penetrated into the support pores, which changes the characteristic of the PP membrane from microporous membrane to a mesoporous material.

3.2.7. Topographical Analysis (Using AFM). Determination of the surface roughness by AFM is crucial to confirm the grafted reaction. PP and modified membranes were analyzed by AFM. The PP has a nodular mesh organized in a framework in which between 5 and 6 fibers converge, allowing the formation of holes with different diameters, and an ovoid geometry was observed. Figure 6 represents (2D) the morphological changes between the PP and the modified membranes (PP-CEL, PP-PS, and PP-CHI). The lightest areas represent the highest regions of the structure and the darkest the deepest. The maximum heights shown for the modified membranes were in the following order: PP-CEL samples $\approx$ $600 \mathrm{~nm}, \mathrm{PP}-\mathrm{CHI} \approx 400 \mathrm{~nm}$, and PP-PS $\approx 300 \mathrm{~nm}$.

The morphology of PP-CEL, likewise PP-PS, showed flakes with a semiradial growth from nodular regions of the PP; these flakes have $54 \%$ more volume. The presence of chitosan promotes the formation of thicker layers, and an amorphous morphology with a soft texture was presented [36]. AFM analysis suggests that the penetration, as well as infiltration of the hybrid materials, is favored in the following order: PP-PS (800 nm), PP-CEL (600 nm), and PPCHI $(300 \mathrm{~nm})$ [37-39].

(1) Surface Roughness. Surface roughness is an important structural property of polymeric membranes. It has been 

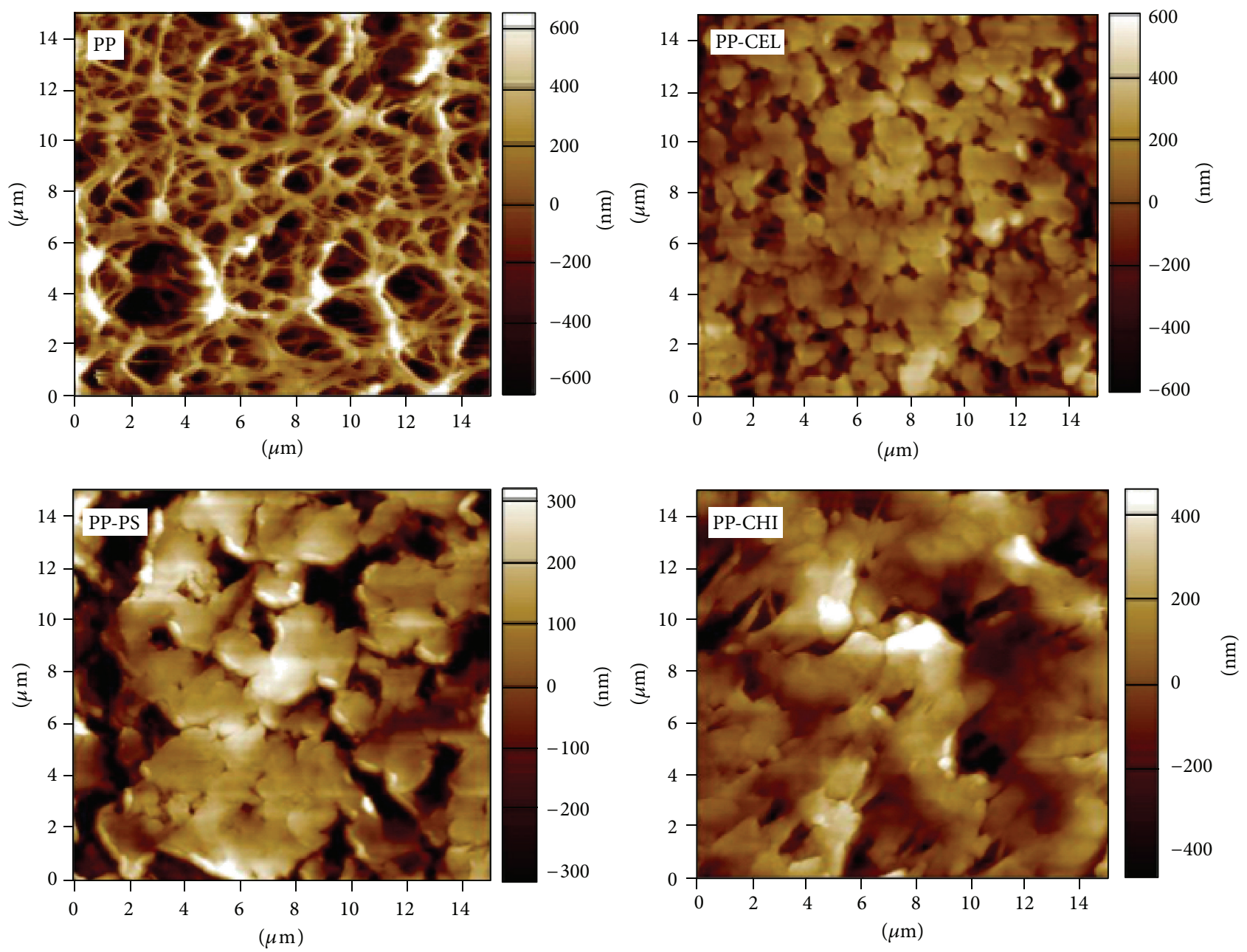

FIGURE 6: AFM topographical image of $15 \times 15$ microns for PP, PP-CEL, PP-PS, and PP-CHI.

TABLE 2: Surface roughness.

\begin{tabular}{lcc}
\hline Membrane & $\begin{array}{c}\text { Roughness (Ra) } \\
\text { Nm }\end{array}$ & $Z$ max (nm) \\
\hline PP & 263.01 & 1700 \\
PP-PS & 212.70 & 1000 \\
PP-CEL & 256.80 & 1400 \\
PP-CHI & 242.59 & 1200 \\
\hline
\end{tabular}

shown that this parameter could be influenced by various membrane fabrication parameters.

Table 2 shows the average of the values of the roughness (Ra) which are related to the penetration and infiltration capability. PP-PS exhibits the lowest roughness; the morphology of the flake has a greater surface and slightly irregular homogeneous characteristics that generate the formation of a film in the regions of greater depth, and the $Z$ values decrease. The PP-CHI presents an intermediate roughness between PP and PP-CEL; the reason is that chitosan film was a hard film and was difficult to penetrate.
TABLE 3: Tensile test of PP and modified membranes.

\begin{tabular}{lccc}
\hline Membrane & $\%$ Gd & Stress (MPa) & Strain (\%) \\
\hline PP & - & 14.1 & 18 \\
PP-CEL & 140 & 56 & 7 \\
PP-PS & 388 & Max. limit & - \\
PP-CHI & 240 & 77.5 & 30 \\
\hline
\end{tabular}

3.2.8. Mechanical Properties Evaluation. The tensile strength and elongation at break for the modified membranes were analyzed [40]. This test determines the main mechanical characteristics of the material and thus indicates its mechanical durability and its plausible application. Table 3 shows the differences in the mechanical properties of each membrane and direct correlation between grafted degree and stress. For the PP-PS membrane the strength at break exceeds $85 \mathrm{MPa}$. This fact indicates that PS gives mechanical stability to PP. The PP-CEL and PP-CHI show an increase of the tensile strength of more than 4 and 5.5 times, respectively, with respect to PP. These results indicate that the modified membranes may be exposed to greater stress. However, the 
TABLE 4: Thermal parameters of PP and modified membranes.

\begin{tabular}{lccccc}
\hline Membrane & $T_{m}\left({ }^{\circ} \mathrm{C}\right)$ & $\Delta H_{m}(\mathrm{~J} / \mathrm{g})$ & $\%$ biopolymer $^{1}$ & $\Delta H_{d 2}(\mathrm{~J} / \mathrm{g})$ & $\mathrm{ND}$ \\
PP & 153.2 & 97.2 & $\mathrm{ND}$ & 165.1 & 680.4 \\
PP-PS & 152.9 & 27.6 & 11.4 & 187.7 \\
PP-CEL & 152.6 & 23.2 & 16.0 & 195.7 \\
PP-CHI & 151.8 & 16.9 & 22.6 & 196.9 & 191.9 \\
\hline
\end{tabular}

${ }^{1}$ Values were obtained from thermogravimetric analysis in a range of $120-300^{\circ} \mathrm{C}$.

percentage of elongation of PP-CEL membrane shows a significant reduction, whereas, for the PP-CHI membrane, this property increases approximately $12 \%$. Therefore, the PP-CHI membranes show significant improvements on their mechanical durability, which potentiate their applications. Not all the surface modifications of the materials improve the mechanical properties. In fact, other reports about the modification of polypropylene, using different methods, showed that the polypropylene decreased its mechanical properties [41].

The improved strength for the three modified membranes confirms that the biopolymer chains were strongly attached to the PP membrane [42] and showed that they could be used in processes that require high resistance to mechanical stress, but with a small elastic capacity.

3.2.9. Thermal Properties and Decomposition Profile. Thermal properties and decomposition profiles of the PP and modified membranes were determined using DSC and TGA. The analysis of the changes in stability and thermal properties of modified membranes is important for their application, as well as the characterization and determination of their chemical and physical changes.

The melting temperatures of grafted membranes and PP were determined from peak onset (Figure 7, peak 1). Heat of melting $\left(\Delta H_{m}\right)$ and heat of decomposition $\left(\Delta H_{d 2}, \Delta H_{d 3}\right)$ of the polymers were determined from the peak area of its corresponding transition in DSC (Figure 7, peaks 2 and 3).

(1) Change in $T_{m}$ and $\Delta H_{m}$. Table 4 shows the values of $T_{m}$ for PP and modified membranes. Fusion temperature was slightly lower for modified membranes than the one of the unmodified PP membrane. This change in $T_{m}$ could be attributed to the crystal arrangement in modified membranes and depends on the grafted biopolymer. The heat of melting $\left(\Delta H_{m}\right)$ for modified membranes decreases with the increase of the total content of biopolymer (\% biopolymer). It has been reported that the thermodynamic melting temperature of semicrystalline biopolymers decreases as the molecular weight decreases and/or as the number of defects increases, that is, with an increase in branching and/or crosslinking [43].

(2) Heat of Decomposition and Natural Polymer Content in Grafted Membranes. In Figure 7, peak 2 refers to an endothermic process associated with decomposition of grafted biopolymer, which was not observed in PP curve. This process was also observed in TGA curves (Figure 8) with evident mass loss in modified membranes at $120-290^{\circ} \mathrm{C}$,

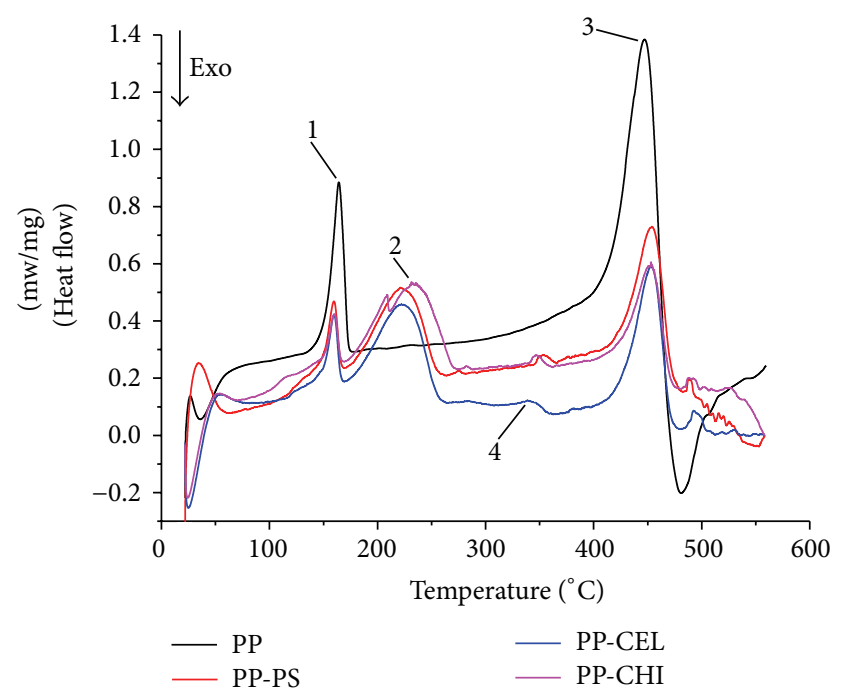

FIGURE 7: DSC curves of PP and modified membranes.

which was associated with a complex process, including dehydration of the biopolymer rings and breaking of the C-O-C glycosidic bonds, present in the main chain of the biopolymer [44]. In addition, Table 4 shows that the largest increase in $\Delta H_{d 2}$ occurs for PP-CHI. It is worth pointing out that this is directly related to the total molar amount of the biopolymer in the membrane and indicates that crosslinking or branching of grafts was greater in PP-CHI.

The second decomposition stage (peak 3 in Figure 7) was related to the PP decomposition, and this peak was observed in the modified membranes as well. The most important characteristic for modified membranes is heat decomposition, $\Delta H_{d 3}$. The energy in modified membranes was lower than that in the PP membrane, and this behavior was observed for the three modified membranes, therefore indicating that grafted polymers require less quantity of energy for decomposition, which would favor their degradation. This process of decomposition was also observed in TGA curves (Figure 8) in $380-480^{\circ} \mathrm{C}$ range. Modified membranes showed a mass loss around $300-380^{\circ} \mathrm{C}$ that can be related to acrylic acid fragments, corroborated by peak 4 in DSC curves (Figure 7).

3.2.10. X-Ray Diffraction Analysis (XRD). In this work the evaluation of the degree of crystallinity of the modified membranes was performed by X-ray diffraction (XRD) [45]. XRD patterns were collected at $2 \theta\left(5-60^{\circ}\right)$, though the most significant differences in the diffraction pattern of the 


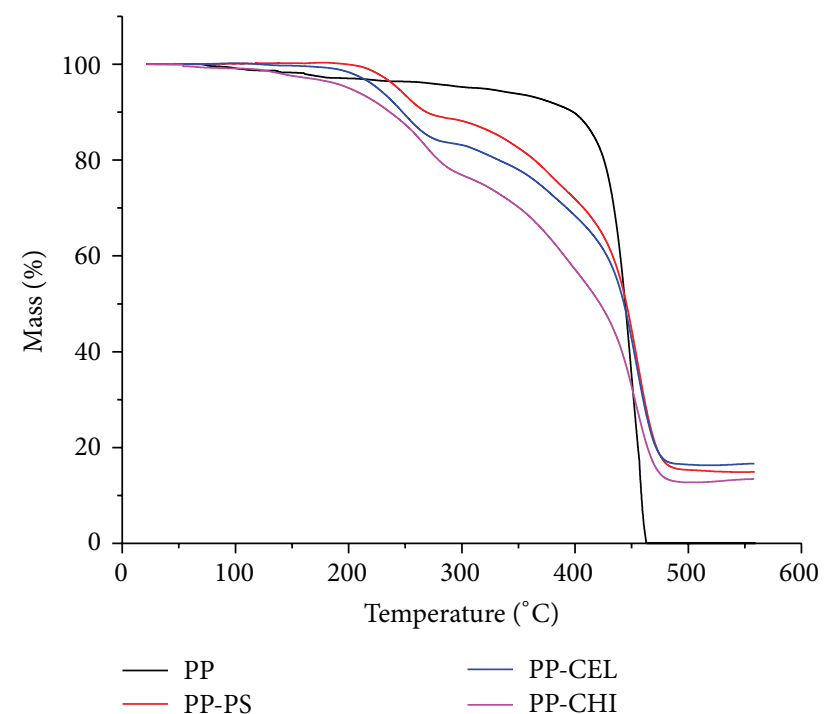

FIGURE 8: TGA curves of polypropylene and modified membranes.

respective phases appear in the range of $10^{\circ}<2 \theta<15^{\circ}$, which directly depends on the grafted biopolymer and was related to the loss of crystallinity in the following order: PP-CEL > PP-PS > PP-CHI (Figure 9). This behavior is probably due to the random arrangement of each grafted biopolymer [46] and the branches formed by graft copolymerization reaction.

3.3. Preliminary Test of Sorption of Copper. The major challenge for the adsorption field is to select the most promising types of sorbent from an extremely large pool of readily available materials. In order to evaluate the capability of removing metal ions of the modified membranes, the PPCHI membrane was selected; it contains polar functional groups such as $-\mathrm{OH}$ and $-\mathrm{NH}_{2}$. These groups can be involved in chemical bonding and are responsible for the capacity for metal ions removal. With this in mind, a preliminary test of sorption process was carried out. $\mathrm{A} \mathrm{Cu}^{+2}$ solution passed through the PP-CHI for 1 hour of operation time and 12 aliquots were collected every $5 \mathrm{~min}$. Experiments were conducted in triplicate. The breakthrough curve shows a tendency to equilibrium after 10 minutes with a value of $C_{e} / C_{i}$ (effluent concentration/initial concentration) equal to 0.8 , indicating that the 80 percent was removed (Figure 10). However, the membrane has not reached saturation point, since the value of $C_{e} / C_{i}$ is different than 1.0.

The copper sorption capacity for the membrane PP-CHI was $35.2 \mathrm{mg} \mathrm{Cu} / \mathrm{g}$; this result indicates that the modified membrane still has active groups for the metal removal. Table 5 shows that the PP-CHI is an excellent alternative for the metal ion removal such as copper comparing with chitosan in different presentation and process conditions [23, 24].

The performed test of copper ions sorption suggests that the modified materials prepared in this work find a potential application in the removal of metal ions.

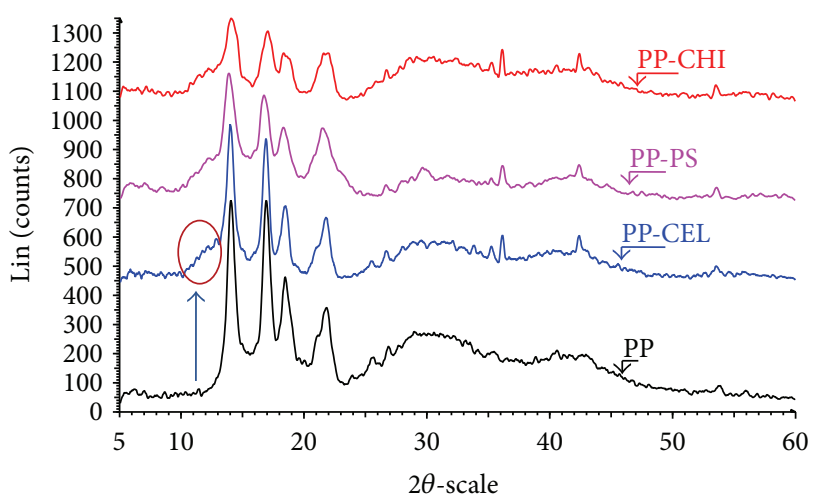

Figure 9: X-ray diffraction analysis of polypropylene (PP) and modified membranes.

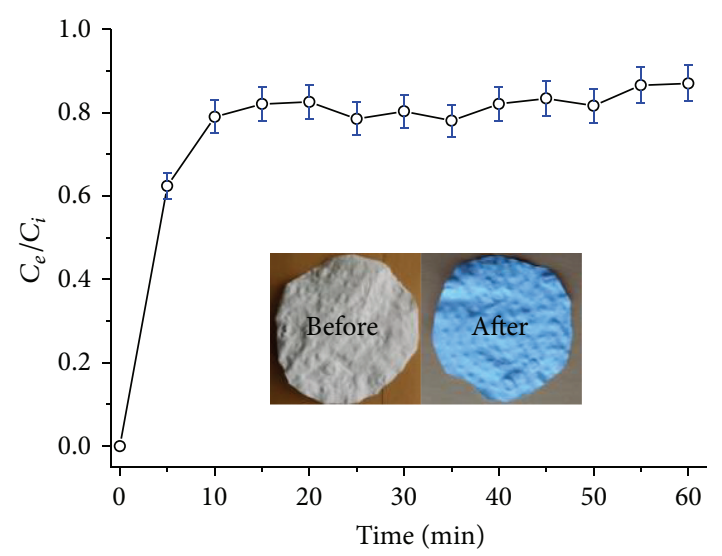

FIGURE 10: Breakthrough curve for sorption of $\mathrm{Cu}^{+2}$ using PP-CHI membrane.

\section{Conclusions}

Three new modified materials were obtained using UV radiation induced graft copolymerization reaction. The use of biopolymers such as potato starch, cellulose, and chitosan resulted in a very good alternative to modify the polypropylene membrane, changing its physical and chemical properties and therefore expanding its applications. The acrylic acid was a good option to functionalize the polypropylene membrane and graft the biopolymers. The grafting reaction depends on natural polymer, reaction time, and concentration. FT-IR-ATR, XRD, TGA, DSC, SEM, $\mathrm{BET}$, and AFM analyses showed that the grafting reaction occurred. The mechanical properties of these membranes were improved. The kinetics of swelling of modified membranes and their water contact angles indicate a complete change to hydrophilic material. The modified membranes could be used for the metal ions removal, due to the affinity of the metal ions with the polar groups present in the modified membranes. 
TABLE 5: Comparative sorbent material for metal ion removal.

\begin{tabular}{lccc}
\hline Sorbent material & $\begin{array}{c}\text { Sorption capacity }(\mathrm{mg} \mathrm{de} \\
\left.\mathrm{Cu}^{2+} / \mathrm{g}\right)\end{array}$ & Process condition & Author \\
\hline Chitosan pearls & $40-200$ & Batch & Flores et al., 2005 [23] \\
Chitosan & $<150$ & Batch & Flores et al., 2005 [23] \\
Chitosan membrane & 0.63 & Continuous & Mora Molina et al., 2012 [24] \\
PP-CHI membrane & 35.2 & Continuous & This work \\
\hline
\end{tabular}

\section{Competing Interests}

The authors declare that they have no competing interests.

\section{Acknowledgments}

The authors would like to thank the Centro Conjunto de Investigación en Química Sustentable UAEM-UNAM and $3 \mathrm{M}$ Company for providing the propylene membrane, Lizbeth Triana Cruz and María Citlalit Martínez Soto for technical assistance, and A. R. Vilchis Nestor for SEM microscopy. Finally, the financial support of SIyEA of UAEMex through Project 3719/2014 and CONACYT through Project 220392 is also acknowledged.

\section{References}

[1] M. Ulbricht, "Advanced functional polymer membranes," Polymer, vol. 47, no. 7, pp. 2217-2262, 2006.

[2] L. H. Sperling and C. E. Carraher Jr., "Renewable resource monomers and polymers," in Polymer Applications of Renewable-Resource Materials, L. H. Sperling and C. E. Carraher Jr., Eds., pp. 1-26, Springer, New York, NY, USA, 1983.

[3] I. Frischinger and S. Dirlikov, "Toughening of epoxy resins by epoxidized soybean oil," in Toughened plastics i, vol. 233, pp. 451-489, American Chemical Society, Washington, DC, USA, 1993.

[4] J. Rösch and R. Mülhaupt, "Toughened polypropylenepolyamide 6 blends prepared by reactive blending," in Toughened Plastics II, vol. 252, pp. 291-302, American Chemical Society, Washington, DC, USA, 1996.

[5] V. Costamagna, M. Strumia, M. López-González, and E. Riande, "Gas transport in surface grafted polypropylene films with poly(acrylic acid) chains," Journal of Polymer Science Part B: Polymer Physics, vol. 45, no. 17, pp. 2421-2431, 2007.

[6] N. H. Taher, A. M. Dessouki, and F. H. Khalil, "Radiation grafting of acrylic acid onto polypropylene films," International Journal of Radiation Applications and Instrumentation Part C: Radiation Physics and Chemistry, vol. 36, no. 6, pp. 785-790, 1990.

[7] M. M. Nasef and E.-S. A. Hegazy, "Preparation and applications of ion exchange membranes by radiation-induced graft copolymerization of polar monomers onto non-polar films," Progress in Polymer Science, vol. 29, no. 6, pp. 499-561, 2004.

[8] I. Pinnau and B. D. Freeman, "Formation and modification of polymeric membranes: overview," in Membrane Formation and Modification, vol. 744 of ACS Symposium Series, chapter 1, pp. 1-22, American Chemical Society, 1999.

[9] T. Matsuura, Synthetic Membranes and Membrane Separation Processes, Taylor \& Francis, New York, NY, USA, 1993.
[10] S. Saxena, A. R. Ray, and B. Gupta, "Chitosan immobilization on polyacrylic acid grafted polypropylene monofilament," Carbohydrate Polymers, vol. 82, no. 4, pp. 1315-1322, 2010.

[11] D. S. Kumar, M. Fujioka, K. Asano, A. Shoji, A. Jayakrishnan, and Y. Yoshida, "Surface modification of poly(ethylene terephthalate) by plasma polymerization of poly(ethylene glycol)," Journal of Materials Science: Materials in Medicine, vol. 18, no. 9, pp. 1831-1835, 2007.

[12] Y. Ogiwara, K. Torikoshi, and H. Kubota, "Vapor phase photografting of acrylic acid on polymer films: effects of solvent mixed with monomer, Journal of Polymer Science: Polymer Letters Edition, vol. 20, no. 1, pp. 17-21, 1982.

[13] A. M. Mika, R. F. Childs, J. M. Dickson, B. E. McCarry, and D. R. Gagnon, "A new class of polyelectrolyte-filled microfiltration membranes with environmentally controlled porosity," Journal of Membrane Science, vol. 108, no. 1-2, pp. 37-56, 1995.

[14] M. E. Cohen, M. A. Grable, and B. M. Riggleman, Second Report on Development of Improved Cellulose Acetate Membranes for Reverse Osmosis, U.S. Dept. of the Interior, Office of Saline Water, Washington, DC, USA, 1970.

[15] M.-X. Hu, Q. Yang, and Z.-K. Xu, "Enhancing the hydrophilicity of polypropylene microporous membranes by the grafting of 2hydroxyethyl methacrylate via a synergistic effect of photoinitiators," Journal of Membrane Science, vol. 285, no. 1-2, pp. 196205, 2006.

[16] Z. Xu, J. Wang, L. Shen, D. Men, and Y. Xu, "Microporous polypropylene hollow fiber membrane: part I. Surface modification by the graft polymerization of acrylic acid," Journal of Membrane Science, vol. 196, no. 2, pp. 221-229, 2002.

[17] A. T. Paulino, M. R. Guilherme, A. V. Reis, G. M. Campese, E. C. Muniz, and J. Nozaki, "Removal of methylene blue dye from an aqueous media using superabsorbent hydrogel supported on modified polysaccharide," Journal of Colloid and Interface Science, vol. 301, no. 1, pp. 55-62, 2006.

[18] Y. Zheng, D. Huang, and A. Wang, "Chitosan-g-poly(acrylic acid) hydrogel with crosslinked polymeric networks for $\mathrm{Ni}^{2+}$ recovery," Analytica Chimica Acta, vol. 687, no. 2, pp. 193-200, 2011.

[19] J. N. BeMiller and R. L. Whistler, Starch: Chemistry and Technology, Elsevier Science, 2009.

[20] P. Jolles and R. A. A. Muzzarelli, Chitin and Chitinases, Springer, Berlin, Germany, 1999.

[21] J. F. Kennedy and L. Quinton, "Column handbook for size exclusion chromatography: Chi-San Wu (Ed.); Academic Press, New York, 1999, 637 pages, ISBN 0-12-765555-7, US\$140.00," Carbohydrate Polymers, vol. 43, no. 2, pp. 205-206, 1999.

[22] R. Lapasin, Rheology of Industrial Polysaccharides: Theory and Applications, Springer US, 2012.

[23] J. A. Flores, A. E. Navarro, K. P. Ramos et al., "Adsorción de CU(II) por quitosano en polvo y perlas de gel," Revista de la Sociedad Química del Perú, vol. 71, pp. 17-25, 2005. 
[24] J. Mora Molina, L. Chaves Barquero, M. Araya Marchena, and R. Starbird Perez, "Chitosan membrane development and design of equipment for the removal of heavy metals from water," Tecnologia en Marcha, vol. 25, no. 3, pp. 3-18, 2012.

[25] C. A. B. Nava-Ortiz, G. Burillo, E. Bucio, and C. AlvarezLorenzo, "Modification of polyethylene films by radiation grafting of glycidyl methacrylate and immobilization of $\beta$ cyclodextrin," Radiation Physics and Chemistry, vol. 78, no. 1, pp. 19-24, 2009.

[26] M. L. Palacios-Jaimes, F. Cortes-Guzman, D. A. GonzálezMartínez, and R. M. Gómez-Espinosa, "Surface modification of polypropylene membrane by acrylate epoxidized soybean oil to be used in water treatment," Journal of Applied Polymer Science, vol. 124, no. 1, pp. E147-E153, 2012.

[27] P. A. Dworjanyn and J. L. Garnett, "Synergistic effects of urea with polyfunctional acrylates for enhancing the photografting of styrene to polypropylene," Journal of Polymer Science Part C: Polymer letters, vol. 26, no. 3, pp. 135-138, 1988.

[28] A. S. Singha, A. Shama, and V. K. Thakur, "Pressure induced graft-co-polymerization of acrylonitrile onto Saccharum cilliare fibre and evaluation of some properties of grafted fibre," Bulletin of Materials Science, vol. 31, no. 1, pp. 7-13, 2008.

[29] S. D. Bhattacharya and M. S. Inamdar, "Polyacrylic acid grafting onto isotactic polypropylene fiber: methods, characterization, and properties," Journal of Applied Polymer Science, vol. 103, no. 2, pp. 1152-1165, 2007.

[30] A. Bhattacharya and B. N. Misra, "Grafting: a versatile means to modify polymers: techniques, factors and applications," Progress in Polymer Science, vol. 29, no. 8, pp. 767-814, 2004.

[31] Z. Xu, L. Wan, and X. Huang, "Techniques for membrane surface characterization," in Surface Engineering of Polymer Membranes, Advanced Topics in Science and Technology in China, chapter 2, pp. 5-63, Springer, Berlin, Germany, 2009.

[32] Z.-K. Xu, X.-J. Huang, and L.-S. Wan, Surface Engineering of Polymer Membranes, Advanced Topics in Science and Technology in China, Springer, Berlin, Germany, 2009.

[33] N. Hameed, S. P. Thomas, R. Abraham, and S. Thomas, "Morphology and contact angle studies of poly(styrene-coacrylonitrile) modified epoxy resin blends and their glass fibre reinforced composites," eXPRESS Polymer Letters, vol. 1, no. 6, pp. 345-355, 2007.

[34] A. Saffar, P. J. Carreau, A. Ajji, and M. R. Kamal, "Development of polypropylene microporous hydrophilic membranes by blending with PP-g-MA and PP-g-AA," Journal of Membrane Science, vol. 462, pp. 50-61, 2014.

[35] R. Jayakumar, M. Prabaharan, R. L. Reis, and J. F. Mano, "Graft copolymerized chitosan-present status and applications," Carbohydrate Polymers, vol. 62, no. 2, pp. 142-158, 2005.

[36] J. M. Bennett, "Recent developments in surface roughness characterization," Measurement Science and Technology, vol. 3, no. 12, pp. 1119-1127, 1992.

[37] L. Palacio, P. Prádanos, A. Hernández, M. J. Ariza, J. Benavente, and M. Nyström, "Phase-contrast scanning force microscopy and chemical heterogeneity of GR polysulfone ultrafiltration membranes," Applied Physics A: Materials Science \& Processing, vol. 73, no. 5, pp. 555-560, 2001.

[38] S. Lowell, J. E. Shields, M. A. Thomas, and M. Thommes, Characterization of Porous Solids and Powders: Surface Area, Pore Size and Density, Springer, Rotterdam, Netherlands, 2012.

[39] M. J. Esplandiu, "Scanning probe microscopies for analytical studies at the nanometer scale," Contributions to Science, vol. 3, pp. 33-46, 2005.
[40] H. Habibi, R. Amrollahi, and M. Alavi, "Polymerization of acrylic acid by a $4 \mathrm{kj}$ plasma focus device," in Proceedings of the 13th International Conference on Plasma Surface Engineering (PSE '12), pp. 270-273, Linköping University Electronic Press, Garmisch-Partenkirchen, Germany, September 2012.

[41] H.-Y. Yu, Z.-K. Xu, H. Lei, M.-X. Hu, and Q. Yang, "Photoinduced graft polymerization of acrylamide on polypropylene microporous membranes for the improvement of antifouling characteristics in a submerged membrane-bioreactor," Separation and Purification Technology, vol. 53, no. 1, pp. 119-125, 2007.

[42] L. A. Fasce, V. Costamagna, V. Pettarin, M. Strumia, and P. M. Frontini, "Poly(acrylic acid) surface grafted polypropylene films: near surface and bulk mechanical response," eXPRESS Polymer Letters, vol. 2, no. 11, pp. 779-790, 2008.

[43] E.-S. A. Hegazy, H. A. A. El-Rehim, and H. A. Shawky, "Investigations and characterization of radiation grafted copolymers for possible practical use in waste water treatment," Radiation Physics and Chemistry, vol. 57, no. 1, pp. 85-95, 2000.

[44] Y. Chen and H.-M. Tan, "Crosslinked carboxymethylchitosang-poly(acrylic acid) copolymer as a novel superabsorbent polymer," Carbohydrate Research, vol. 341, no. 7, pp. 887-896, 2006.

[45] S. Kavesh and J. M. Schultz, "Meaning and measurement of crystallinity in polymers: a review," Polymer Engineering \& Science, vol. 9, no. 6, pp. 452-460, 1969.

[46] P. Zugenmaier, Crystalline Cellulose and Derivatives: Characterization and Structures, Springer, Berlin, Germany, 2008. 

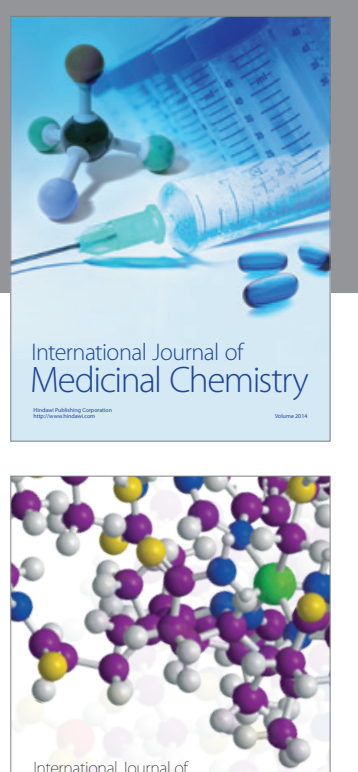

Carbohydrate Chemistry

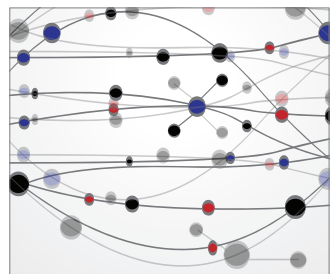

The Scientific World Journal
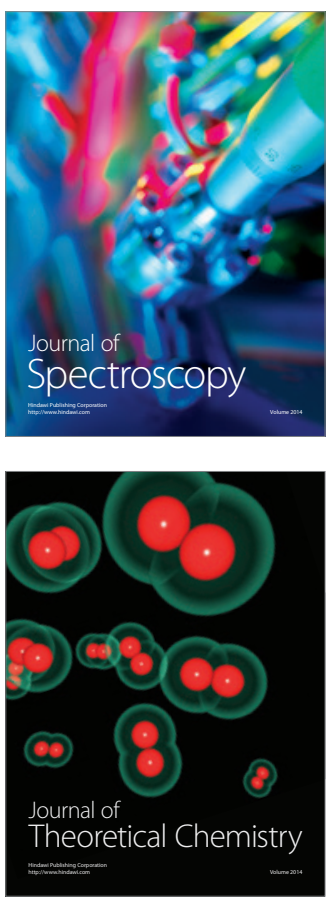
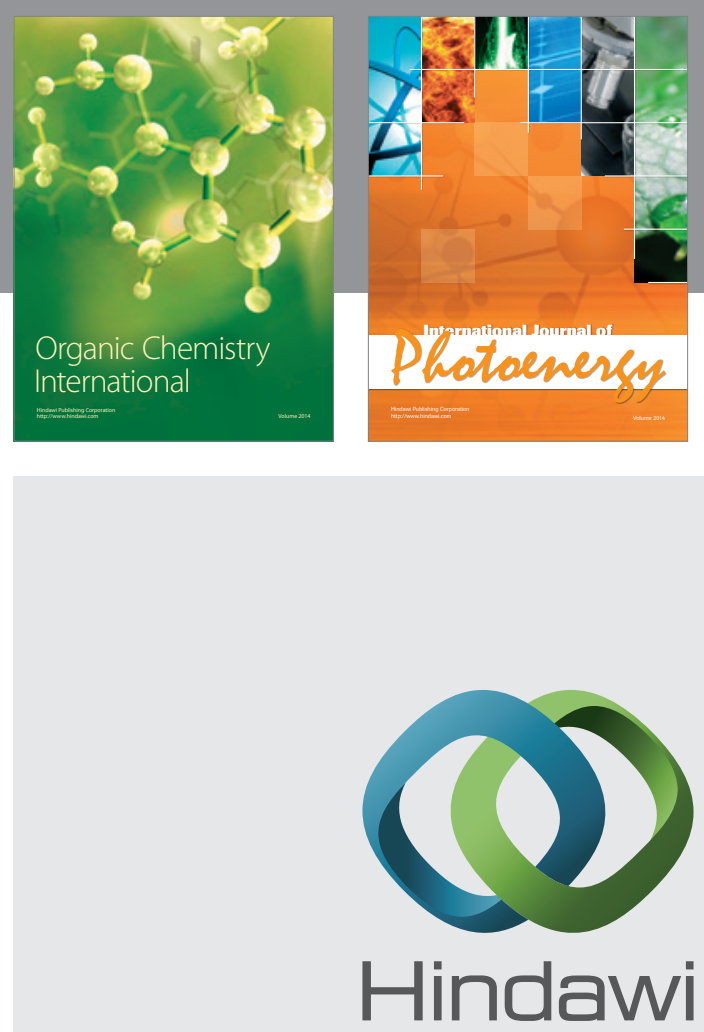

Submit your manuscripts at

http://www.hindawi.com

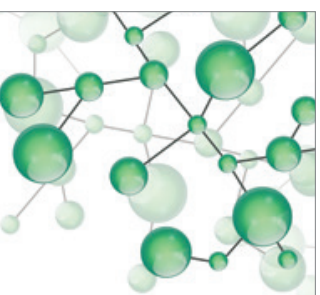

International Journal of

Inorganic Chemistry

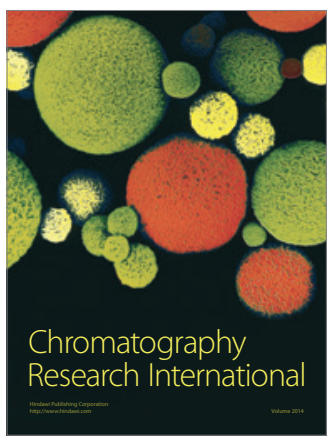

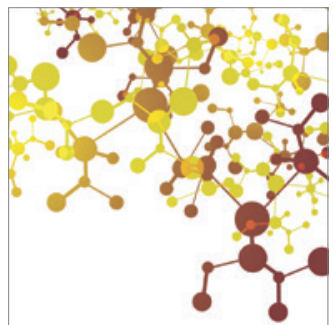

Applied Chemistry
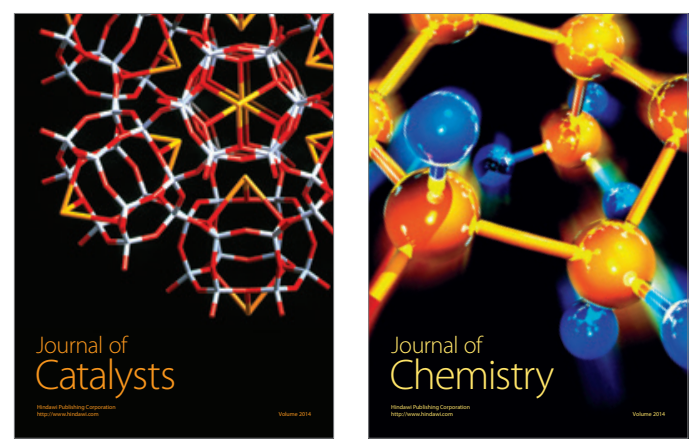
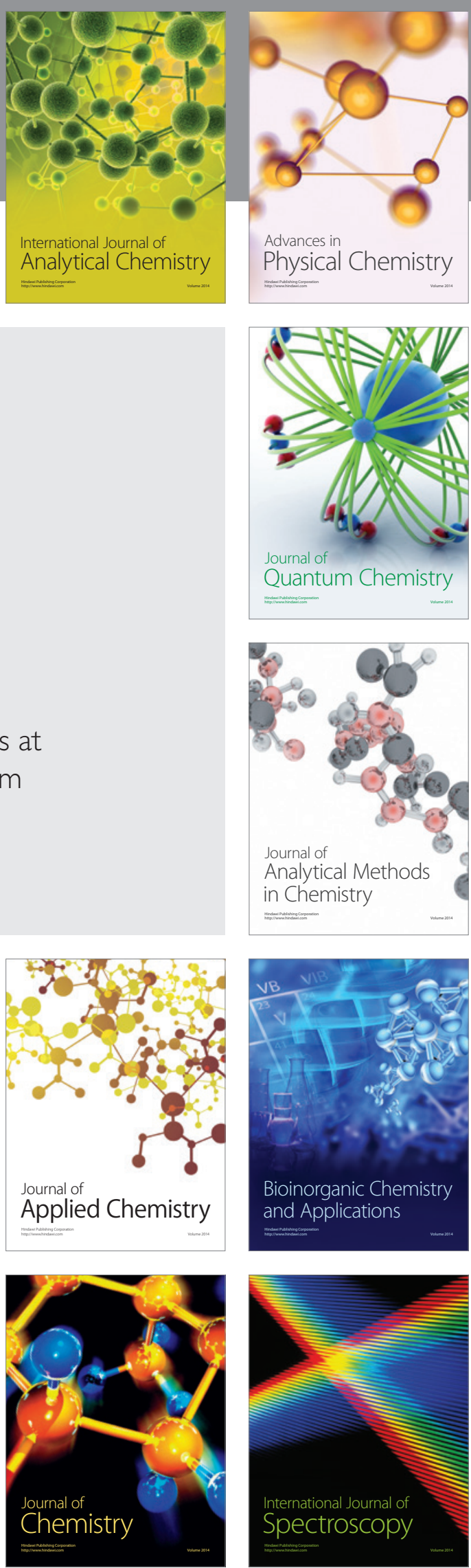